Article

\title{
Non-Polar Wurtzite (112̄0) GaN/AlN Quantum Dots for Highly Efficient Opto-Electronic Devices
}

\author{
Seoung-Hwan Park ${ }^{1}$ and Doyeol Ahn ${ }^{2,3, *}$ \\ 1 Department of Electronics Engineering, Catholic University of Daegu, Hayang, \\ Kyeongsan 38430, Gyeongbuk, Korea; shpark@cu.ac.kr \\ 2 Department of Electrical and Computer Engineering, University of Seoul, Seoulsiripdae-ro 163, \\ Tongdaimoon-Gu, Seoul 02504, Korea \\ 3 Physics Department, Charles E Schmidt College of Science, Florida Atlantic University, \\ Boca Raton, FL 33431-0991, USA \\ * Correspondence: dahn@uos.ac.kr
}

Received: 3 June 2020; Accepted: 1 August 2020; Published: 5 August 2020

\begin{abstract}
In III-nitride quantum dots (QDs), optical transition rate is very low because of the large built-in electrostatic field caused by the spontaneous polarization (SP) and piezoelectric (PZ) effects. In this work, we study the screening potential which is a solution of the self-consistent Hartree equation taking into account the built-in electrostatic field and its effect on light emission characteristics of non-polar wurtzite (WZ) (112̄0) GaN/AlN QD. It is found that the light emission intensity of the non-polar (1120) GaN/AlN QD structure is expected to be about four times larger than that of the c-plane (0001) GaN/AlN QD structure because the $y$-polarized matrix elements in the non-polar QD are larger than that in the c-plane QD. These predictions indicate that non-polar GaN/AIN QD structure have strong potential for highly efficient opto-electronic devices.
\end{abstract}

Keywords: GaN; AlN; quantum dot; non-polar; light emission

\section{Introduction}

Wurtzite (WZ) GaN-based quantum dots (QDs) have attracted significant attention as promising candidates for application in optical, optoelectronic, and electronic devices [1-7]. Unfortunately, these structures have large spontaneous (SP) electric dipoles along the [0001] direction (c-axis) of the lattice on top of strong piezoelectric (PZ) polarization [8,9], which results in the substantial reduction of transition probability between the electrons and holes in heterostructures.

To overcome these problems, nanostructures along nonpolar directions have been proposed by several authors. In the case of quantum well $(\mathrm{QW})$ structures, the electric field is shown to vanish when they are grown on the non-polar substrate $[10,11]$. On the other hand, in the case of QD structures, facets along the [0001] direction still remain even when grown on a non-polar substrate because they are three dimensional objects, in contrast to the case of non-polar QWs. Therefore, consideration of the screening effect on the internal field due to SP and PZ polarizations will be important for the design of non-polar QD devices [12,13]. There has been very little work done on studies on the light emission characteristics of nonpolar QD structures taking into account the screening potential effects self-consistently.

In this paper, we investigate screening potential arising from the internal field and light emission characteristics of non-polar WZ (112̄0) GaN/AlN QDs by using $k \cdot p$ multi-band effective mass theory. The wavefunctions are obtained by solving the Schrödinger equation for electrons and the $(11 \overline{2} 0)$ $6 \times 6$ Hamiltonian for holes $[14,15]$ adopted for the QD structure. We consider a cubic QD structure $(\mathrm{GaN})$ grown on $\mathrm{GaN}$ of a length $d$, which is embedded in AlN cladding material with a size of 
$200 \times 200 \times 200 \AA^{3}$ ). In Figure 1, we plotted schematic geometry for (a) c-plane and (b) a-plane QD structures. The growth axes for c-plane and a-plane correspond to (0001)- and (112̄0)-orientations.

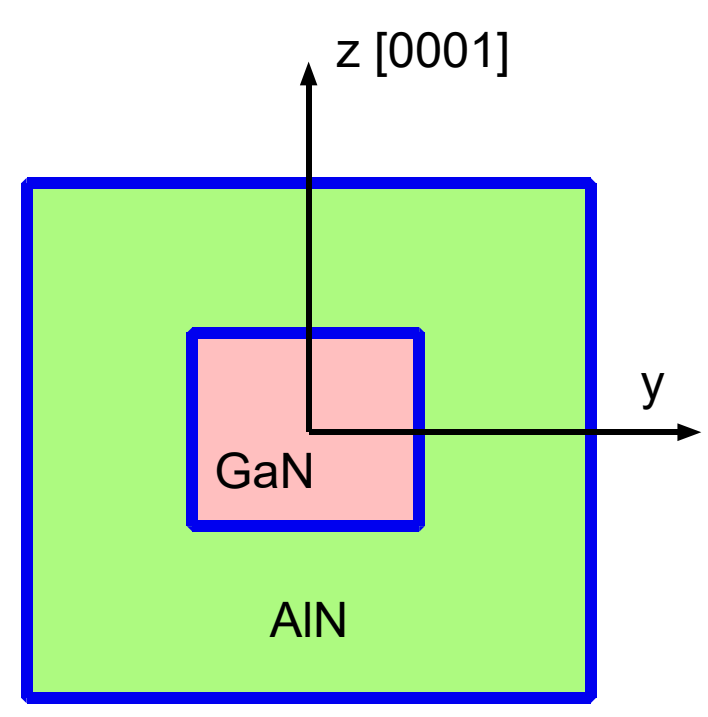

(a) c-plane

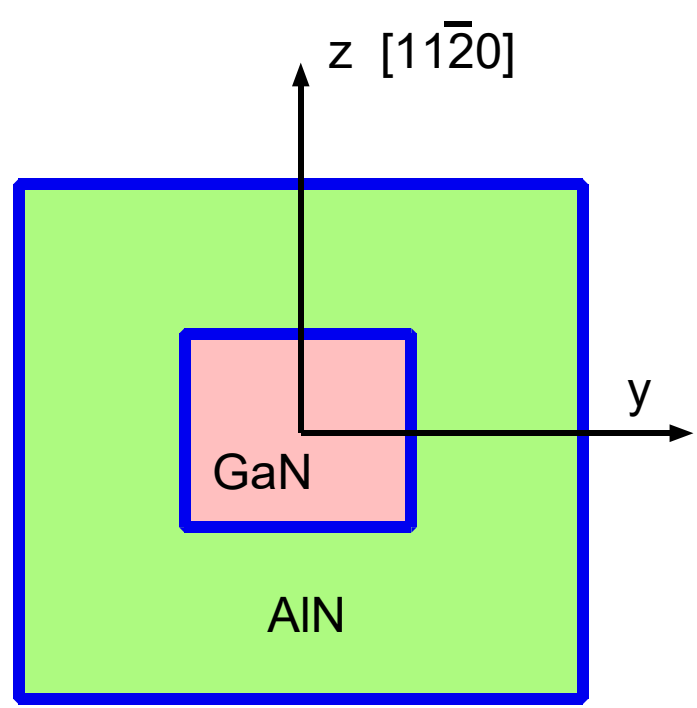

(b) a-plane

Figure 1. Schematic geometry for (a) c-plane and (b) a-plane QD structures.

\section{Theory}

\section{1. (0001)-Oriented Hamiltonian and Built-in Potential}

The (0001)-oriented c-plane Hamiltonian for the valence-band structure can be written as $[14,15]$

$$
H(\mathbf{k}, \epsilon)=\left(\begin{array}{cccccc|l}
F & -K^{*} & -H^{*} & 0 & 0 & 0 \\
-K & G & H & 0 & 0 & \Delta & \mid U_{1}> \\
-H & H^{*} & \lambda & 0 & \Delta & 0 & \mid U_{2}> \\
0 & 0 & 0 & F & -K & H & \mid U_{3}> \\
0 & 0 & \Delta & -K^{*} & G & -H^{*} \\
0 & \Delta & 0 & H^{*} & -H & \lambda
\end{array}\right) \mid \begin{aligned}
& \mid U_{5}> \\
& \mid U_{6}>
\end{aligned}
$$

where

$$
\begin{aligned}
F= & \Delta_{1}+\Delta_{2}+\lambda+\theta \\
G= & \Delta_{1}-\Delta_{2}+\lambda+\theta \\
\lambda= & \frac{\hbar^{2}}{2 m_{0}}\left[A_{1} k_{z}^{2}+A_{2}\left(k_{x}{ }^{2}+k_{y}{ }^{2}\right)\right]+D_{1} \epsilon_{z z}+D_{2}\left(\epsilon_{x x}+\epsilon_{y y}\right) \\
\theta= & \frac{\hbar^{2}}{2 m_{0}}\left[A_{3} k_{z}^{2}+A_{4}\left(k_{x}{ }^{2}+k_{y}{ }^{2}\right)\right]+D_{3} \epsilon_{z z}+D_{4}\left(\epsilon_{x x}+\epsilon_{y y}\right) \\
K= & \frac{\hbar^{2}}{2 m_{o}} A_{5}\left(k_{x}+i k_{y}\right)^{2} \\
H= & \frac{\hbar^{2}}{2 m_{o}} A_{6}\left(k_{x}+i k_{y}\right) k_{z} \\
& \Delta=\sqrt{2} \Delta_{3}
\end{aligned}
$$


Here, $\Delta_{1}$ is the crystal-field split energy, $\Delta_{2}$ and $\Delta_{3}$ account for spin-orbit interactions, the $A_{i}{ }^{\prime} \mathrm{s}$ are the valence-band effective-mass parameters, the $D_{i}$ 's are the deformation potentials for wurtzite crystals, $k_{i}$ is the wave vector, and $\epsilon_{i j}$ is the strain tensor. Here, $\epsilon_{x x}=\left(a_{s}-a_{e}\right) / a_{e}$ and $\epsilon_{z z}=\left(c_{s}-c_{e}\right) / c_{e}$, which are due to mismatches between the lattice constants of the well $\left(a_{e}\right.$ and $\left.c_{e}\right)$ and the substrate $\left(a_{s}\right.$ and $c_{s}$ ). The bases for the Hamiltonian are defined as

$$
\begin{aligned}
& \left|U_{1}>=-\frac{1}{\sqrt{2}}\right|(X+i Y) \uparrow>, \\
& \left|U_{2}>=\frac{1}{\sqrt{2}}\right|(X-i Y) \uparrow>, \\
& \left|U_{3}>=\right| Z \uparrow>, \\
& \left|U_{4}>=\frac{1}{\sqrt{2}}\right|(X-i Y) \downarrow>, \\
& \left|U_{5}>=-\frac{1}{\sqrt{2}}\right|(X+i Y) \downarrow>, \\
& \left|U_{6}>=\right| Z \downarrow>.
\end{aligned}
$$

The strain tensors and built-in potential are obtained by [16]

$$
\begin{gathered}
\sigma_{i j}=C_{i j l m} \epsilon_{i j}-e_{k j i} E_{k}, \\
D_{i}=\epsilon_{0} \epsilon_{r} E_{j}+P_{i}+P_{s p},
\end{gathered}
$$

where $\sigma$ are vectors of the stress, $C$ is the elastic stiffness tensor, $e$ is the piezoelectric constant tensor, and $D$ is the electric flux, which are related to the strain $\epsilon$ and electric field $E$ vectors. Here, $P_{i}, \epsilon_{r}$, and $P_{s p}$ are the piezoelectric polarization, relative dielectric constants, and spontaneous polarization, respectively.

\section{2. (112̄0)-Oriented-Oriented Hamiltonian}

The Hamiltonian for an arbitrary crystal orientation can be obtained using a rotation matrix

$$
U=\left(\begin{array}{ccc}
\cos \theta \cos \phi & \cos \theta \sin \phi & -\sin \theta \\
-\sin \phi & \cos \phi & 0 \\
\sin \theta \cos \phi & \sin \theta \sin \phi & \cos \theta
\end{array}\right)
$$

Rotations of the Euler angles $\theta$ and $\phi$ transform the physical quantities from $(x, y, z)$ coordinates to $\left(x^{\prime}, y^{\prime}, z^{\prime}\right)$ coordinates. The $z$-axis corresponds to the $c$-axis [0001]. The relation between the coordinate systems for vectors and tensors is expressed as [17-19]

$$
\begin{aligned}
k_{p} & =U_{i p} k_{i \prime}^{\prime} \\
\epsilon_{p q} & =U_{i p} U_{j q} \epsilon_{i j}^{\prime} \\
e_{p q r} & =U_{i p} U_{j q} U_{j r} e_{i j k}^{\prime} \\
C_{p q r s} & =U_{i p} U_{j q} U_{j r} U_{j s} C_{i j k}^{\prime}
\end{aligned}
$$

where summation over repeated indices is indicated. The a-plane Hamiltonian with a set of faces $\{11 \overline{2} 0\}$ can be obtained by substituting $\phi=0$ and $\theta=\pi / 2$ into Equation (1). The misfit strain, $\epsilon_{0}$, of the QD is taken with respect to the surrounding matrix and is assumed to be equal to $\left(2 \epsilon_{0 a}+\epsilon_{0 c}\right) / 3$, where $\epsilon_{0 a}$ is the misfit strain in the $x-y$ plane and $\epsilon_{0 c}$ is the misfit strain along the $z$ plane [20]. 


\subsection{Self-Consistent Calculation}

The total potential profiles for the electrons and holes are [21]

$$
\begin{aligned}
& V_{c}(\mathbf{r})=V_{c b}(\mathbf{r})+V(\mathbf{r})-|e| \phi(\mathbf{r}) \\
& V_{v}(\mathbf{r})=V_{v b}(\mathbf{r})-V(\mathbf{r})+|e| \phi(\mathbf{r}),
\end{aligned}
$$

where $V_{c b}$ and $V_{v b}$ are the square potential for the conduction band and valence band, respectively, $V$ is the static electric potential induced by the piezoelectric and spontaneous polarizations, and $\phi$ is the screening potential induced by the charged carriers, which satisfies Poisson's equation

$$
\nabla \cdot(\epsilon(\mathbf{r}) \nabla \phi(\mathbf{r}))=-|e|[p(\mathbf{r})-n(\mathbf{r})],
$$

where $\epsilon$ is the dielectric constant. The density of state for a quantum dot is given by $\rho(E)=\delta(E-$ $\left.E_{n, m}\right) / V$, where $V$ is the volume of the QD. Then, the quasi-Fermi levels for a given carrier density are calculated from the charge neutrality using the following relations [16]:

$$
\begin{aligned}
& n=\frac{2}{V} \sum_{n} \frac{1}{1+\exp \left[\frac{\left(E_{n}-E_{f c}\right)}{k T}\right]} \\
& p=\frac{1}{V} \sum_{m} \frac{1}{1+\exp \left[\frac{\left(E_{m}-E_{f v}\right)}{k T}\right]},
\end{aligned}
$$

where $E_{f c}$ and $E_{f v}$ are the relevant quasi-Fermi levels. Here, we assumed that carriers are injected into the QD and the contribution from continuum states is negligible in Equation (12). The electron and hole concentrations, $p(\mathbf{r})$ and $n(\mathbf{r})$, in Equation (11) are related to the wavefunctions of the $n$-th conduction subband and the $m$-th valence subband by [16]

$$
n(\mathbf{r})=2 \sum_{n}\left|\phi_{n}(\mathbf{r})\right|^{2} \frac{1}{1+\exp \left[\frac{\left(E_{n}-E_{f c}\right)}{k T}\right]}
$$

and

$$
p(\mathbf{r})=\sum_{m} \sum_{v=1}^{6}\left|g_{m}^{(v)}(\mathbf{r})\right|^{2} \frac{1}{1+\exp \left[\frac{\left(E_{m}-E_{f v}\right)}{k T}\right]}
$$

where $v$ refers to the bases for the Hamiltonian, $\phi_{n}(\mathbf{r})$ and $g_{m}^{(v)}(\mathbf{r})(v=1,2,3,4,5$, and 6) are envelope functions in the conduction and valence bands, respectively.

\subsection{Optical Matrix Element and Spontaneous Emission Coefficient}

The optical momentum matrix elements $\mathbf{M}$ for the QD are given by [15]

$$
|\hat{\mathbf{e}} \cdot \mathbf{M}|^{2}=\left|<\Psi_{c}^{n}\right| \hat{\mathbf{e}} \cdot \mathbf{p}\left|\Psi_{h}^{m}>\right|^{2},
$$

where $\Psi_{c}$ and $\Psi_{h}$ are the wave functions for the conduction and the valence bands, respectively. The indices $n=\left\{n_{1} n_{2} n_{3}\right\}$ and $m=\left\{m_{1} m_{2} m_{3}\right\}$ denote the electron states in the conduction band and the subband states in the valence band, respectively. The polarization-dependent interband momentum-matrix elements can be written as 
TE -polarization $(\hat{\mathbf{e}}=\cos \phi \hat{\mathbf{x}}+\sin \phi \hat{\mathbf{y}})$ :

$$
\begin{aligned}
\left|\hat{\mathbf{e}} \cdot \mathbf{M}^{\uparrow}\right|^{2} & =\mid \cos \phi\left\{-\frac{1}{\sqrt{2}} P_{x}\left\langle g_{m}^{(1)} \mid \phi_{n}\right\rangle+\frac{1}{\sqrt{2}} P_{x}\left\langle g_{m}^{(2)} \mid \phi_{n}\right\rangle\right\} \\
& +\left.\sin \phi\left\{-i \frac{1}{\sqrt{2}} P_{x}\left\langle g_{m}^{(1)} \mid \phi_{n}\right\rangle-i \frac{1}{\sqrt{2}} P_{x}\left\langle g_{m}^{(2)} \mid \phi_{n}\right\rangle\right\}\right|^{2} \\
\left|\hat{\mathbf{e}} \cdot \mathbf{M}^{\downarrow}\right|^{2} & =\mid \cos \phi\left\{\frac{1}{\sqrt{2}} P_{x}\left\langle g_{m}^{(4)} \mid \phi_{n}\right\rangle-\frac{1}{\sqrt{2}} P_{x}\left\langle g_{m}^{(5)} \mid \phi_{n}\right\rangle\left|\phi_{n}\right\rangle\right\} \\
& +\left.\sin \phi\left\{-i \frac{1}{\sqrt{2}} P_{x}\left\langle g_{m}^{(4)} \mid \phi_{n}\right\rangle-i \frac{1}{\sqrt{2}} P_{x}\left\langle g_{m}^{(5)} \mid \phi_{n}\right\rangle\right\}\right|^{2}
\end{aligned}
$$

$\mathrm{TM}$-polarization $(\hat{\mathbf{e}}=\hat{\mathbf{z}})$ :

$$
\begin{aligned}
\left|\hat{\mathbf{e}} \cdot \mathbf{M}^{\uparrow}\right|^{2} & =\left|P_{z}\left\langle g_{m}^{(3)} \mid \phi_{n}\right\rangle\right|^{2} \\
\left|\hat{\mathbf{e}} \cdot \mathbf{M}^{\downarrow}\right|^{2} & =\left|P_{z}\left\langle g_{m}^{(3)} \mid \phi_{n}\right\rangle\right|^{2} .
\end{aligned}
$$

Furthermore,

$$
\begin{aligned}
P_{x} & =P_{y}=\left\langle S\left|p_{x}\right| X\right\rangle=\left\langle S\left|p_{y}\right| Y\right\rangle=\frac{m_{o}}{\hbar} P_{2}, P_{z}=\left\langle S\left|p_{z}\right| Z\right\rangle=\frac{m_{o}}{\hbar} P_{1}, \\
P_{1}^{2} & =\frac{\hbar^{2}}{2 m_{o}}\left(\frac{m_{o}}{m_{e}^{z}}-1\right) \frac{\left(E_{g}+\Delta_{1}+\Delta_{2}\right)\left(E_{g}+2 \Delta_{2}\right)-2 \Delta_{3}^{2}}{E_{g}+2 \Delta_{2}}, \\
P_{2}^{2} & =\frac{\hbar^{2}}{2 m_{o}}\left(\frac{m_{o}}{m_{e}^{t}}-1\right) \frac{E_{g}\left[\left(E_{g}+\Delta_{1}+\Delta_{2}\right)\left(E_{g}+2 \Delta_{2}\right)-2 \Delta_{3}^{2}\right]}{\left(E_{g}+\Delta_{1}+\Delta_{2}\right)\left(E_{g}+\Delta_{2}\right)-\Delta_{3}^{2}} .
\end{aligned}
$$

The spontaneous emission coefficient for the quantum dot is [1]

$$
\begin{aligned}
g_{s p}(\hbar \omega)= & \frac{e^{2}}{m_{o}^{2} \omega} \sqrt{\frac{\mu_{o}}{\epsilon}} \sum_{n, m} \frac{1}{V}\left|\hat{\mathbf{e}} \cdot \mathbf{M}_{m}^{n}\right|^{2} \\
& \cdot \frac{\hbar / \tau_{i n}}{\left(E_{h m}^{e n}-\hbar \omega\right)^{2}+\left(\hbar / \tau_{i n}\right)^{2}}\left(f_{c}^{n}-f_{v}^{m}\right),
\end{aligned}
$$

where $m_{0}$ is the free-electron mass, $\omega$ is the angular frequency, $\mu_{0}$ is the vacuum permeability, and $\tau_{\text {in }}$ is the intraband relaxation time, which is assumed to be $1 \times 10^{-13} \mathrm{~s}$.

\section{Results}

Figure 2 shows the calculated potential along $x$-, $y$-, and $z$-axes for (a) (0001)- and (b) (1120)-oriented GaN/AIN QD structures grown on GaN substrate. The length $d$ of cubic QD is set to be $40 \AA$. The (0001)-oriented GaN/AIN QD structure shows that there exists a large potential along $x-, y-$, and $z$-axes. The potentials along $x$ - and $y$-axes are mainly due to the strain-induced piezoelectric polarization. In the case of the potential along $z$-axis, its magnitude is observed to be slightly larger than that along $x$ - or $y$-axis because there exists a spontaneous polarization along $z$-axis. On the other hand, in the case of the (112̄0)-oriented GaN/AIN QD structure, the dominant potential is observed for the case along $x$-axis only because the spontaneous polarization is toward the polar [0001] direction and the strain-induced piezoelectric polarization is small. As a result, we expect that the internal field effect can be reduced for the non-polar GaN/AIN QD structure. 

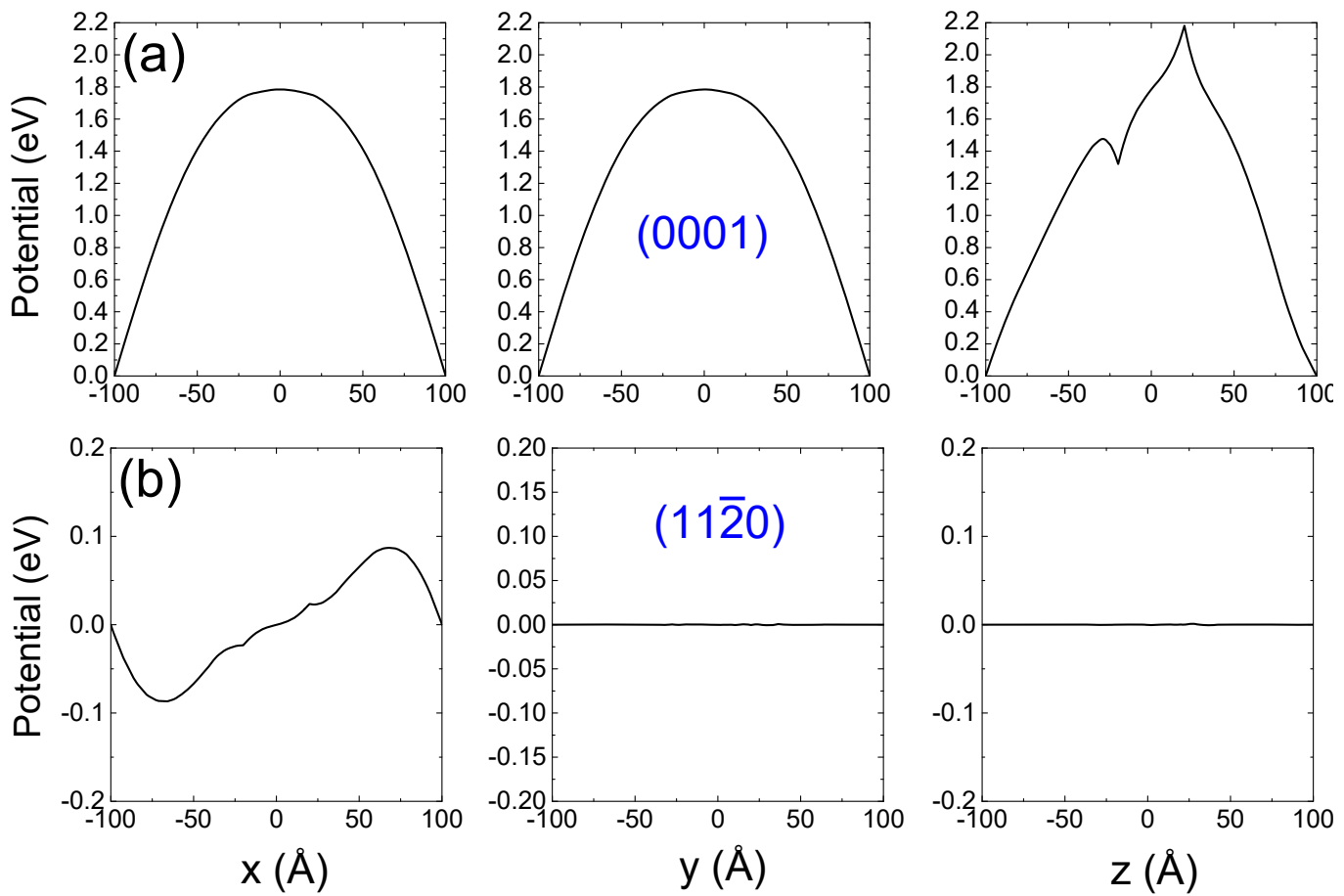

Figure 2. Calculated potential along $x-, y-$, and $z$-axes for (a) (0001)- and (b) (1120)-oriented GaN/AlN QD structures grown on GaN substrate. The length $d$ of cubic QD is set to be $40 \AA$.

Figure 3 shows the screening potential $\phi(\mathbf{r})$ along $x$-, $y$-, and $z$-axes for (a) (0001)- and (b) (1120)-oriented a-plane GaN/AlN QD structures grown on GaN substrate. The (0001)-oriented GaN/AlN QD structure shows that the screening potential along $z$-axis is larger than that along $x$ - or $y$-axis because there exists larger internal field along $z$-axis due to the spontaneous polarization. In the case of the (1120)0)-oriented GaN/AIN QD structure, however, dominant screening potential is observed along $x$-axis while the screening potential along $y$ - or $z$-axis is relatively small. The magnitude of the screening potential may be related to the magnitude of the potential due to the PZ and SP polarizations. Furthermore, the increase in the screening potential will affect the optical matrix elements.

Figure 4 shows the normalized optical matrix element for $x-, y$-, and $z$-polarization for (a) (0001)and (b) (1120)-oriented a-plane GaN/AIN QD structures grown on GaN substrate. The $x$ or $y$ coordinate indicates the subband index in the conduction and valence bands. $(x, y)=(i, j)$ means that the matrix element corresponds to the transition between $i$-th subband in the conduction band and $j$-th subband in the valence band. The quasi-Fermi levels in the conduction and valence bands are located below the first subband for both (0001)- and (1120)-oriented QD structures. That is, energy spacings between the quasi-Fermi energy and the first subband are -0.020 and $-0.042 \mathrm{eV}$ for the conduction and valence bands in the (0001)-oriented QD structure, respectively. Similar results are obtained for the (1120)-oriented QD structure. Thus, in the case of the conduction band, we plotted only two subbands because the energy spacing between subbands is large $(\sim 0.24 \mathrm{eV})$. On the other hand, in the case of the valence band, we plotted twelve subbands because the energy spacings between subbands are very small $(\sim 0.005)$. 

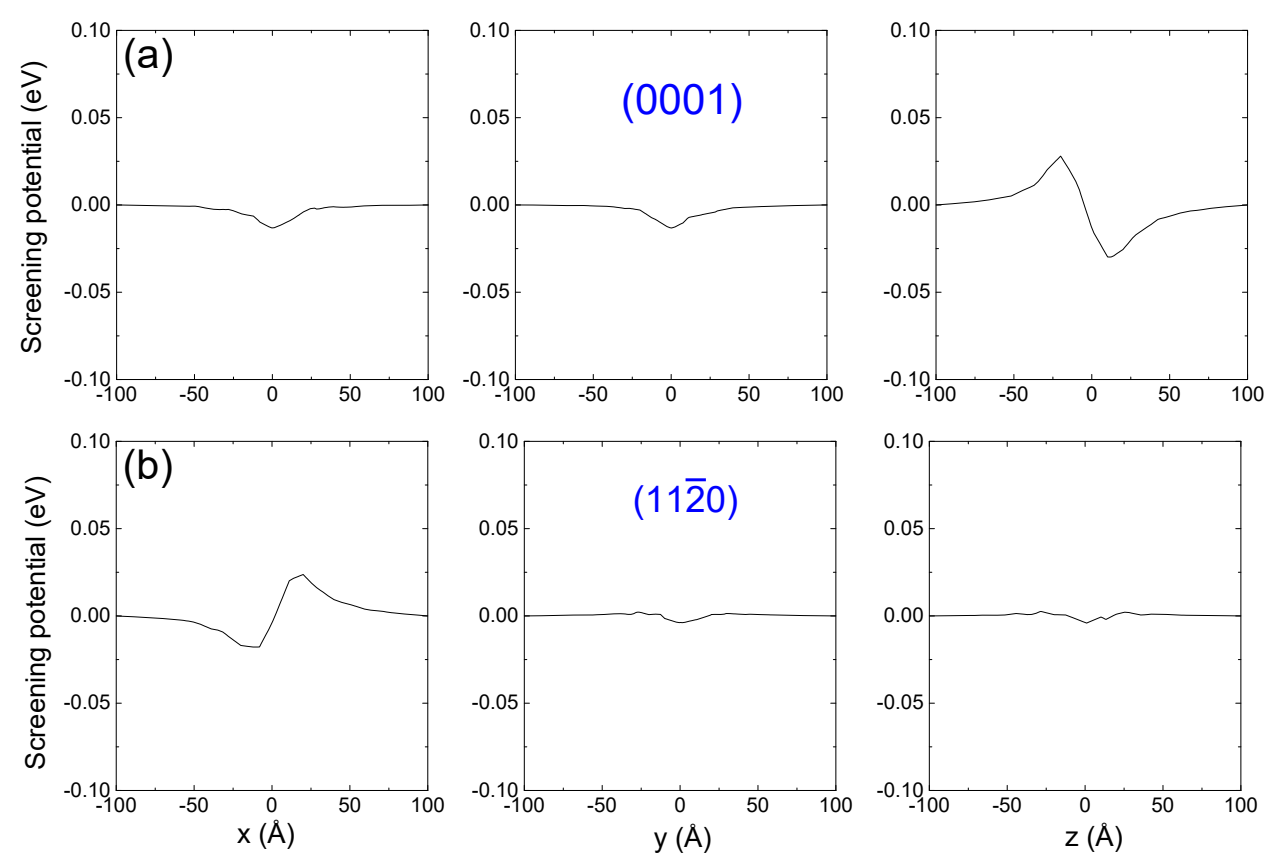

Figure 3. Screening potential $\phi(\mathbf{r})$ along $x-, y$-, and $z$-axes for (a) (0001)- and (b) (112̄0)-oriented a-plane $\mathrm{GaN} / \mathrm{AlN}$ QD structures grown on GaN substrate.

In the case of the $x$-polarization, the (0001)-oriented GaN/AlN QD structure shows that dominant matrix elements are related to transition between the first subband in conduction and the first two valence bands. On the other hand, the (1120)-oriented QD structure shows that the matrix elements related to transition between the first subband in conduction and the 4- and 5-th valence bands are dominant and larger than those of the (0001)-oriented GaN/AIN QD structure. However, the (0001)-oriented GaN/AIN QD structure shows that $x$-polarized light intensity is larger than that of the (1120)-oriented QD structure. This means that matrix elements related to the transition between lower subbands are important because carrier population in higher subbands is low. On the other hand, the (1120)-oriented GaN/AIN QD structure shows much larger $y$-polarized matrix elements than the (0001)-oriented GaN/AIN QD structure, which is related to the transition between lower subbands. Thus, the $y$-polarized light intensity for the GaN/AlN QD structure is about four times larger than that for the (0001)-oriented GaN/AlN QD structure. Similar tendency is observed for the case of the $z$-polarization.

Figure 5 shows the spontaneous emission spectra for $x-, y$-, and $z$-polarizations for (0001)- and (1120)-oriented a-plane GaN/AlN QD structures grown on GaN substrate. Spontaneous emission coefficients are calculated at the carrier density of $\mathrm{N}_{3 D}=1 \times 10^{19} \mathrm{~cm}^{-3}$. The (0001)-oriented GaN/AlN QD structure shows that $x$ - or $y$-polarized light intensity is much larger than $z$-polarized light intensity. This is because the optical matrix element for $x$ - or $y$-polarization between ground state subbands is relatively larger than that for the z-polarization. On the other hand, the (1120)-oriented GaN/AlN QD structure shows that the light intensity for $y$-polarization is dominant. Furthermore, we observe that the $y$-or $z$-polarized light intensity for the (1120)-oriented GaN/AIN QD structure is four times larger than that for the (0001)-oriented GaN/AlN QD structure. Thus, we expect that non-polar GaN/AlN QD structure can be used for optoelectonic device with high light intensity, as observed for QW structures. 

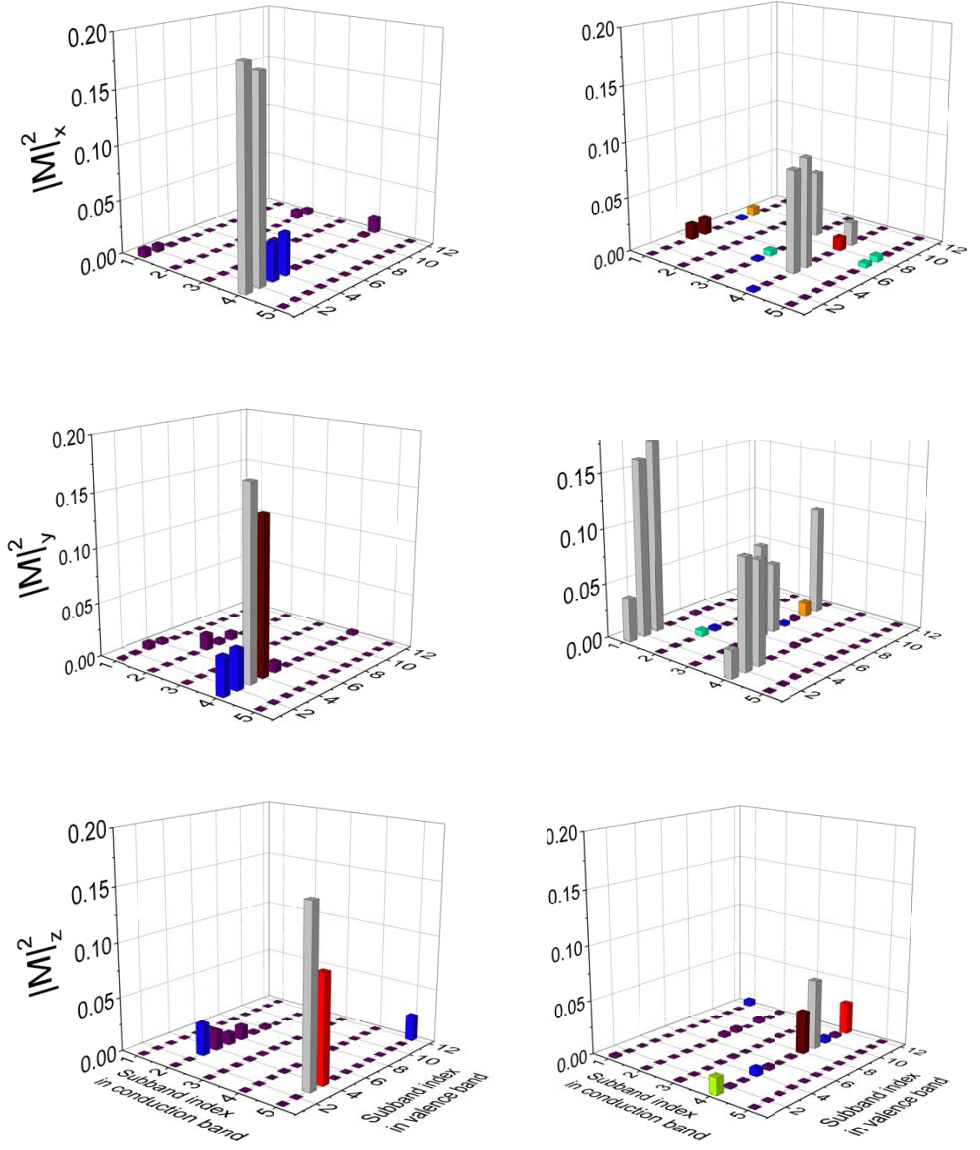

(a) $(0001)$

(b) $(11 \overline{2} 0)$

Figure 4. Normalized optical matrix element for $x$-, $y$-, and $z$-polarization for (a) (0001)- and (b) (112̄0)-oriented a-plane GaN/AIN QD structures grown on GaN substrate.
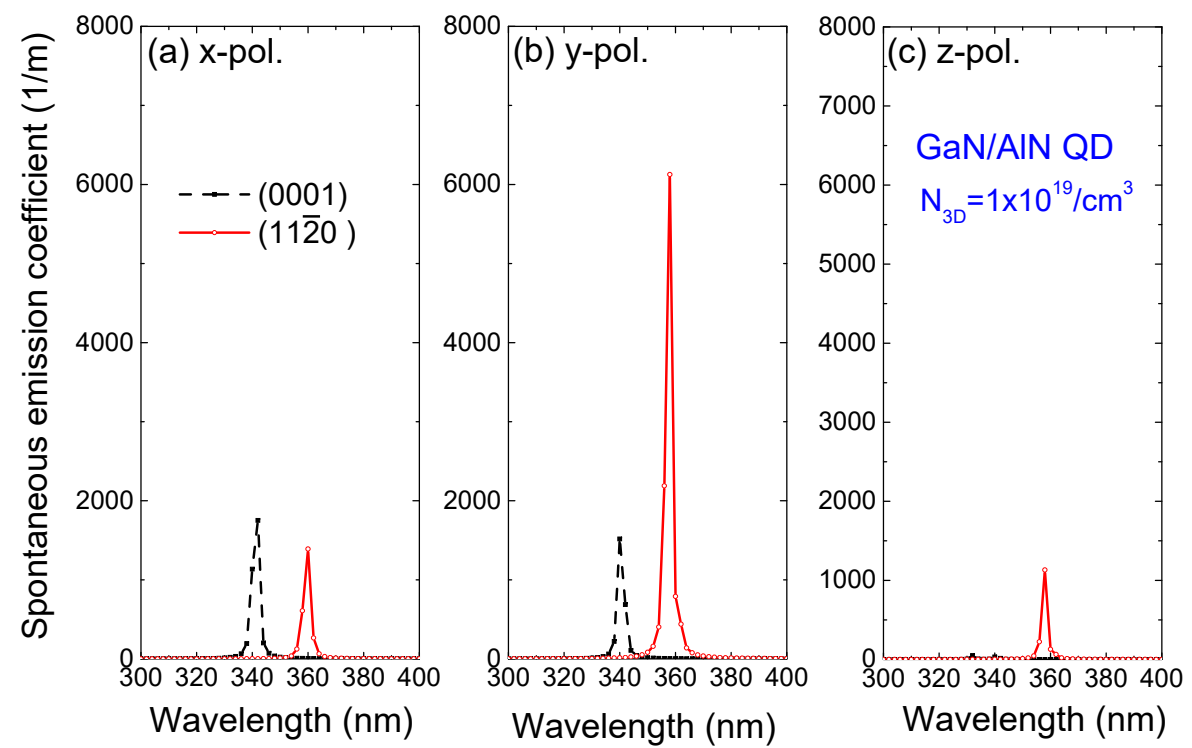

Figure 5. Spontaneous emission spectra for $x-, y$-, and $z$-polarizations for (0001)- and (112̄0)-oriented a-plane GaN/AlN QD structures grown on GaN substrate. 


\section{Conclusions}

We investigated screening potential and light emission characteristics of non-polar WZ GaN/AlN QDs by using an effective mass theory. The (0001)-oriented GaN/AIN QD structure shows that the screening potential along $z$-axis is larger than that along $x$ - or $y$-axis because there exists larger internal field along $z$-axis. In the case of the (1120)-oriented GaN/AlN QD structure, however, the screening potential along $x$-axis is large while the screening potential along $y$-or $z$-axis is very small. The light intensity for the a-plane GaN/AIN QD structure is shown to be about four times larger than that for the (0001)-oriented GaN/AIN QD structure because the internal field of the former is reduced, compared to that of the latter. We expect that non-polar GaN/AIN QD structure can be used for optoelectonic device with high light intensity, as observed for QW structures.

Author Contributions: S.-H.P. contributed software and data calculations and D.A. contributed writing and editing. All authors have read and agreed to the published version of the manuscript.

Funding: S.-H.P. was supported by Basic Science Research Program through the National Research Foundation of Korea(NRF) funded by the Ministry of Education, Science and Technology(2018R1D1A1B07042028). D. Ahn is supported by the 2017 Research Grant from the University of Seoul.

Conflicts of Interest: There is no conflict of interest.

\section{References}

1. Bimberg, D.; Grundmann, M.; Ledentsov, N.N. Quantum Dot Heterostructure; Wiley: New York, NY, USA, 1999.

2. Marquardt, O.; Hickel, T.; Neugebauer, J. Polarization-induced charge carrier separation in polar and nonpolar grown GaN quantum dots. J. Appl. Phys. 2009, 106, 083707. [CrossRef]

3. Schulz, S.; Berube, A.; O’Reilly, E.P. Polarization fields in nitride-based quantum dots grown on nonpolar substrates. Phys. Rev. B 2009, 79, 081401. [CrossRef]

4. Schulz, S.; O'Reilly, E.P. Built-in fields in non-polar $I n_{x} G a_{1-x} N$ quantum dots. Phys. Status Solidi C 2010, 7 80. [CrossRef]

5. Caro, M.A.; Schulz, S.; Healy, S.; O'Reilly, E.P. Built-in field control in alloyed c-plane III-N quantum dots and wells. J. Appl. Phys. 2011, 109, 084110. [CrossRef]

6. Young, T.D.; Jurczak, G.; Lotsari, A.; Dimitrakopulos, G.P.; Komninou, P.; Dłużewski, P. A study of the piezoelectric properties of semipolar $11 \overline{2} 2 \mathrm{GaN} / \mathrm{AlN}$ quantum dots. Phys. Status Solidi B 2015, 252 2296-2303. [CrossRef]

7. Budagosky, J.A.; Garro, N.; Cros, A.; García-Cristóbal, A.; Fount, S.; Daudin, B. Optical properties of wurtzite GaN/AlN quantum dots grown on non-polar planes: The effect of stacking faults in the reduction of the internal electric field. Mater. Sci. Semicond. Process. 2016, 49, 76-80. [CrossRef]

8. Bernardini, F.; Fiorentini, V.; Vanderbilt, D. Spontaneous polarization and piezoelectric constants of III-V nitrides. Phys. Rev. B 1997, 56, 10024. [CrossRef]

9. Martin, G.; Botchkarev, A.; Rockett, A.; Morkoc, H. Valence-band discontinuities of wurtzite GaN, AlN, and InN heterojunctions measured by x-ray photoemission spectroscopy. Appl. Phys. Lett. 1996, 68, 2541. [CrossRef]

10. Park, S.-H. Crystal orientation effects on electronic properties of Wurtzite GaN/AlGaN quantum wells with spontaneous and piezoelectric polarization. Jpn. J. Appl. Phys. 2000, 39, 3478. [CrossRef]

11. Waltereit, P.; Brandt, O.; Trampert, A.; Grahn, H.T.; Menniger, J.; Ramsteiner, M.; Reiche, M.; Ploog, K.H. Nitride semiconductors free of electrostatic fields for efficient white light-emitting diodes. Nature 2000, 406, 865. [CrossRef] [PubMed]

12. Su, G.L.; Frost, T.; Bhattacharya, P.; Dallesasse, J.M.; Chuang, S.L. Detailed model for the $\operatorname{In}_{0.18} \mathrm{Ga}_{0.82} \mathrm{~N} / \mathrm{GaN}$ self-assembled quantum dot active material for $\lambda=420 \mathrm{~nm}$ emission. Opt. Express 2014, 22, 22716. [CrossRef] [PubMed]

13. Park, S.-H.; Ahn, D. Screening effects on the optical properties of II-VI wurtzite $\mathrm{ZnO} / \mathrm{MgO}$ quantum dots. Phys. Rev. B 2018, 97, 075301. [CrossRef]

14. Chuang, S.L.; Chang, C.S. $k \cdot p$ method for strained wurtzite semiconductors. Phys. Rev. B 1996, 54, 2491. [CrossRef] [PubMed] 
15. Park, S.-H.; Chuang, S.L. Crystal-orientation effects on the piezoelectric field and electronic properties of strained wurtzite semiconductors. Phys. Rev. B 1999, 59, 4725. [CrossRef]

16. Boxberg, F.; Tulkki, J. Theory of the electronic structure and carrier dynamics of strain-induced (Ga, In)As quantum dots. Rep. Prog. Phys. 2007, 70, 1425. [CrossRef]

17. Hinckley, J.M.; Singh, J. Influence of substrate composition and crystallographic orientation on the band structure of pseudomorphic Si-Ge alloy films. Phys. Rev. B 1990, 42, 3546. [CrossRef] [PubMed]

18. Nye, J.F. Physical Properties of Crystals; Clarendon: Oxford, UK, 1989.

19. Hong, K.B.; Kuo, M.K. Effect of piezoelectric constants in electronic structures of InGaN quantum dots. Semicond. Sci. Technol. 2013, 28, 105006. [CrossRef]

20. Williams, D.P.; Andreev, A.D.; O’Reilly, E.P. ; Faux, D.A. Derivation of built-in polarization potentials in nitride-based semiconductor quantum dots. Phys. Rev. B 2005, 72, 235318. [CrossRef]

21. Park, S.-H.; Chuang, S.L. Piezoelectric effects on electrical and optical properties of wurtzite GaN/AlGaN quantum well lasers. Appl. Phys. Lett. 1998, 72, 3103. [CrossRef]

(C) 2020 by the authors. Licensee MDPI, Basel, Switzerland. This article is an open access article distributed under the terms and conditions of the Creative Commons Attribution (CC BY) license (http://creativecommons.org/licenses/by/4.0/). 